\title{
智能电网中 OTN 网络规划及组网探讨
}

\section{Discussion on the OTN Internet Planning and Networking in Smart Power Grids}

汤辉 ${ }^{1}$ 韩振连 $^{2}$

Hui Tang ${ }^{1}$ Zhenlian $\operatorname{Han}^{2}$

\author{
1.南瑞集团有限公司(国网电力科学研究 \\ 院有限公司） \\ 中国・江苏 南京 210009 ; \\ 2. 国网江苏电力有限公司沛县供电分公司 \\ 中国・江苏徐州 221600 \\ 1. NARI Group(State Grid Electric Power Research \\ Institute Co.,Ltd.), \\ Nanjing, Jiangsu, 210009, China; \\ 2. State Grid Jiangsu Electric Power Co.,Ltd., \\ Peixian Power Supply Branch, \\ Xuzhou, Jiangsu, 221600, China
}

【摘要】论文分析智能电网中所需的业务, 对 OTN 网络规划及组网进行探讨, 以期实现 智能电网通信网络的逐渐融合, 保证其应用前景的明朗。

【Abstract】This paper analyzes the business needed in smart grid, and discusses OTN network planning and networking, so as to realize the gradual integration of smart grid communication network and ensure its bright application prospect.

【关键词】智能电网; OTN;OLP;ODUk

【Keywords】smart grid; OTN; OLP; ODUk

【DOI】10.36012/etr.v1i2.251

\section{1 引言}

近 10 年来, 在电力系统中光传输系统主要 SDH 和 WDM 组网为主, SDH 主要实现最高带宽 10G 速率的组网, 带宽资源有限; 而 WDM 则主要完成点对点的大容量传输, 但 DWM 缺乏灵活的管理能力。随着智能电网的蓬勃发展, 其相关业务需求也突飞猛进，大颗粒带宽 (2.5G/10G/40G/ $100 \mathrm{G}$ )的需求越来越多,传统的 SDH 和 DWDM 技术已经满 足不了智能电网高带宽的需求, 由此面向大带宽、大颗粒的 OTN 技术应运而生 ${ }^{[1]}$ 。

光传送网 OTN (Optical Transport Network) 是一种新的光 传输技术, 它分为光层和电层体系结构, 对于光层和电层的各 层都有相应的管理监控机制 ${ }^{[2]}$ 。OTN 技术从 SDH/MSTP 技术 体制发展而来，把 SDH/MSTP 的网络可靠性高、保护机制完 善、OAM 功能丰富、组网灵活性强这些优点应用到 WDM 系 统中, 让 OTN 技术既具备了 SDH/MSTP 安全、稳定、可靠, 又 具备了 WDM 的容量大特点。
针对智能电网大颗粒业务和 IP 业务多的特点, OTN 技术 有着良好的先天性优势。支持多种业务接人不同颗粒度的业 务在不同层面统一交换; 灵活的光电交叉,可实现高效的业务 调度; 完备的保护体现结构全方位提高网络可靠性; 简化的网 管端到端业务部署, 充分利用 OTN 的技术特点, 对现有电力 通信骨干传输网进行升级改造, 使电力传输网骨干网向大容 量、可管理、灵活组网、稳定安全的通信网络发展。

\section{2 电力通信网现有业务分析}

\section{1 业务类型}

电力通信的业务类型主要包含生产调度系统、管理信息 化系统和电力营销业务系统。

\section{1 .1 生产调度业务系统}

生产调度业务系统包括电力系统专有业务和调度系统业 务两类。其中,调度系统业务包括实时的 SCADA/EMS 系统、 保护运行信息管理系统及安稳运行管理信息系统、电能量计 量系统等数据业务。 


\section{1 .2 管理信息化业务系统}

管理信息化业务主要包含财务管理系统、ERP 系统、项 目管理系统、人资管理系统、办公自动化系统及安全生产管理 系统等业务。智能电网和互联网系统日益增加的信息化数据 对通信网的带宽及安全性都提出了更高要求, 必须提供可靠、 安全、高带宽的业务通道。

\subsection{3 电力营销业务系统}

电力营销业务系统是通过配网通信及自动化技术，实现 电力用户用电数据采集实时化、业务处理信息化等, 它是智能 化电网中的一个重要组成部分。

\section{2 电力通信业务系统现有带宽分析}

现有的通信业务的承载形式主要以 TDM 业务和 IP 业务 为主,业务的带宽主要如表 1 所示。

表 1 业务带宽

\begin{tabular}{c|c|c}
\hline 业务类型 & 业务带宽 & 备注 \\
\hline 调度数据网 & $2 \mathrm{Mbit} / \mathrm{s}$ & $\mathrm{TDM} / \mathrm{SDH}$ 业务 \\
数据通信网 & $4 \times 155 \mathrm{Mbit} / \mathrm{s}$ & $\mathrm{IP}$ 业务 \\
综合数据网 & $4 \times 155 \mathrm{Mbit} / \mathrm{s}$ & $\mathrm{IP}$ 业务 \\
其他 & $\mathrm{N} \times 155 \mathrm{Mbit} / \mathrm{s}$ & $\mathrm{IP}$ 业务 \\
\hline
\end{tabular}

\section{3 带宽瓶颈}

依照《国网调控中心十三五规划》的要求,数据通信网及 调度数据网均需通过传输网承载 ${ }^{[3]}$ 。数据通信网带宽需求为 省 $10 \mathrm{G}$ 通道,调度数据网二平面带宽提升至千兆级 (1Gbps), 一平面带宽全面提升至 $155 \mathrm{Mbps}$ 以上, 省以上节点逐步提升 至千兆级 $(1 \mathrm{Gbps})$ 。省地接入网核心、汇聚节点间带宽提升至 $155 \mathrm{Mbps}$ 以上。

省级骨干传输网随着业务带宽需求的不断增加，采用 OTN 传输通信网, 承载大颗粒度的生产管理类业务传送的需 求, 覆盖网省公司、 $500 \mathrm{kV}$ 变电站、地市公司及 $220 \mathrm{kV}$ 站点 等。经测算,省公司至所辖地市公司因业务发展需求和基础设 施现状等因素带宽需求差异较大,一般在 10Gbit/s, 部分断面 业务流量超过 10Gbit/s, 结合网络拓扑分析, 传输系统容量需 求为 10 20Gbit/s。现有传输网带宽需求已超出了现有 SDH 传 输网络的承载能力和单波长传输系统的技术极限。

\section{3 规划目标}

2018 年底, 国网开始在电力系统打造全新的泛在互联 网, 泛在互联网和之前的智能电网都是作为推进能源互联网 的重要举措, 意在通过大数据、云计算、物联网共同构建一个 全新的电网。泛在互联网和智能电网的大数据和云计算对与 带宽量和质量要求都很高, 调度数据网、数据通信网的大带宽 链路带宽都在升级改造计划中，部分上联通道带宽要达到万 兆级和千兆级, 这对目前网络带宽为 $10 \mathrm{G}$ 的 SDH 传输承载网
络提出了新的挑战。国网公司各网省骨干传输网按基于 SDH 技术体制 SW-A 平面和基于 OTN 技术体制 SW-B 平面的双 平面架构经行建设, 其中 $\mathrm{SW}-\mathrm{B}$ 平面带宽为 $40 \times 10 \mathrm{~Gb} / \mathrm{s}$ 。以满 足未来大容量业务承载、解决部分超长距跨段传送、释放现网 光缆纤芯资源为建设目标, 建设成一张以承载网省电力生产 III IV 区非实时类大颗粒业务为主的省级通信骨干 OTN 传 输网。

\section{OTN 规划策略}

\subsection{OTN 保护方案}

$\mathrm{OTN}$ 技术的保护分为光层和电层两类。包括基于光层的 光线路保护 (OLP) 和光通道保护, 以及基于电层的 SNCP 保 护和环网保护。

根据相关数据测算, 在光层保护过程中, 信号在光缆中的 传输时延大约是 $0.005 \mathrm{~ms} / \mathrm{km}$, 在电层保护过程中, 信号在单 套 OTN 设备内部线路板至线路板间的时延大约是 $0.3 \mathrm{~ms}$, 在 线路板至支路板的时延大约是 $0.1 \mathrm{~ms}$, 即单套设备的驻留时 延大约是 $0.4 \mathrm{~ms}$ 。以保护路径为 $1000 \mathrm{~km}$, 通过站点 15 个来计 算, 信号传输的总时延大约是 $0.005 \times 1000+0.4 \times 15=11 \mathrm{~ms}$ 。在 电力 OTN 项目中, 部分骨干长距离跨环业务的传输时延可能 达到十几 ms, 但由于 OTN 承载的业务基本都是分组类的业 务, 因此,在传输时延方面都能满足要求。

\section{1 .1 光层保护方案}

基于光层的保护主要有光线路保护( OLP)和光通道保护 的 2 种保护, OTU 板内 $1+1$ 保护与客户侧 $1+1$ 保护 ${ }^{[4]}$ 。光线路 保护 (OLP)利用光保护类单板 OLP 的双发选收功能, 在比邻 网元间利用两条独立的路由对光纤线路进行保护，主备倒换 依据光功率进行。OTU 板内 $1+1$ 保护同样利用 OUT 单板或者 光保护类单板 OLP、DCP 的双发选收功能, 利用分离路由方 式对业务进行保护。客户侧 $1+1$ 保护运用光保护单板的双发 选收功能, 在 OUT 线路故障、板卡故障、子框故障情况下对业 务进行保护。

\section{表 2 OTN 光层保护方案比较}

\begin{tabular}{|c|c|c|c|}
\hline 描述 & 光线路保护 & 板内 $1+1$ 保护 & 客户侧 $1+1$ 保护 \\
\hline 保护范围 & 线路光纤 & Och 路径 & OUT 单板、Och 路径 \\
\hline 应用场景 & 链形组网 & $\begin{array}{c}\text { 环形或则网状行组 } \\
\text { 网 }\end{array}$ & 所有组网型式 \\
\hline 保护特点 & $\begin{array}{l}\text { 1.线路耦合器 } \\
\text { 双发、光开关 } \\
\text { 选收; } 2 \text {. 保护 } \\
\text { 线路光纤支 } \\
\text { 持分段保护; } \\
\text { 3.简单稳定 }\end{array}$ & $\begin{array}{l}\text { 1.OUT 线路光层双 } \\
\text { 发、电层选收; } 2 \text {. 通 } \\
\text { 过对线路备份的方 } \\
\text { 式,提供对 Och 光纤 } \\
\text { 进行保护; } 3 \text {. 成本低 }\end{array}$ & $\begin{array}{l}\text { 1.OUT 客户光层双发 } \\
\text { 选收; } 2 \text {. 通过占用工 } \\
\text { 作及保护两个波长, } \\
\text { 采用不同的路由进行 } \\
\text { 传输的方式, 对 OUT } \\
\text { 单板和 Och 光纤进 } \\
\text { 行保护; } 3 \text {. 应用时间 } \\
\text { 长, 保护范围广 }\end{array}$ \\
\hline
\end{tabular}




\section{1 .2 电层保护方案}

电层保护主要包括 ODUk SNCP 保护、SW SNCP 保护、支 路 SNCP 保护和 ODUk 环网保护。

ODUk SNCP 保护利用电层交叉的双发选收进行保护, 交 叉粒度为 ODUk。对线路板, PID 单板和 OCh 光纤上传输的业 务进行保护。SW SNCP 保护运用单板的电层交叉的双发选收 功能, 对 OCh 通道和线路板进行保护, 交叉粒度为 GE 或 Any 业务。支路 SNCP 保护利用电层交叉的双发选收进行保护, 交 叉粒度为 ODUk, 对支路接入的客户侧 SDH/SONET 或 OTN 业务进行保护。ODUk 环网保护通过占用 2 个不同的 ODUk 通道实现对所有站点间多条分布式业务的保护, 保护通道被 环上各个区段的工作通道共享。

\section{表 3 OTN 电层保护方案比较}

\begin{tabular}{c|c|c|c|c}
\hline 描述 & ODUk SNCP & SW SNCP & 支路 SNCP & ODUk 环网 \\
\hline 工作原理 & $\begin{array}{c}\text { 双发选收 } \\
\text { 单端倒换 }\end{array}$ & $\begin{array}{c}\text { 双发选收 } \\
\text { 单端倒换 }\end{array}$ & $\begin{array}{c}\text { 双发选收 } \\
\text { 单端倒换 }\end{array}$ & $\begin{array}{c}\text { 单发单收 } \\
\text { 双端倒换 }\end{array}$ \\
\hline 交叉颗粒 & ODUk & GE、Any & ODUk & ODUk \\
\hline 保护范围 & $\begin{array}{c}\text { 保护线路板,PID } \\
\text { 单板和 OCh 光 } \\
\text { 纤上传输的业务护 OCh 通 }\end{array}$ & $\begin{array}{l}\text { 保护支线路板和接人 } \\
\text { TOM 板 }\end{array}$ & $\begin{array}{l}\text { 的客户侧 SDH } \\
\text { 或 OTN 业条护相邻站 }\end{array}$ \\
APS间的业务 \\
\hline AP协议 & 不需要 & 不需要 & 不需要 & 需要 \\
\hline
\end{tabular}

\subsection{OTN 系统配置原则}

第一, 在电力通信 OTN 网络中现主要承载 GE、10G 等不 同颗粒 IP 业务, 对本站上下业务及穿通业务都进行光电光转 换处理, 能够灵活实现对业务的调度及再生, 并使得信号的性 能达到最佳, 从而合理规避了 OSNR 过差对系统造成的恶劣 影响, 同时解决了业务跨环、跨段点较多时承载的问题, 所以 宜采用电交叉技术。

第二, 由于设计围绕电层交叉理念, 因此, 设备的交叉连 接矩阵板容量应充分考虑业务发展的需要, 具备较强的平滑 升级及保护功能。

第三, OTN 波长转换单元板卡尽量配置为支线路分离 型。同时考虑设备母板的安全性, OTU 板件应根据方向来设 置, 保证每个 OTU 板件只面向唯一的方向, 且不同光方向的 波道位于不同母板之上。

第四, 根据网络结构, 合理配置站点光方向的数量, 按照 一个光方向一个光层子架的原则配置。某些特殊站点, 如跨域 连接, 可以多配置光层子架, 满足未来的需要。

第五, 系统配置应考虑维护使用和扩容的需要, 具备 $80 \times$ $10 \mathrm{Gbit} / \mathrm{s}$ 平滑升级的功能, 同时应满足 $10 \mathrm{Gbit} / \mathrm{s}$ 和 $40 \mathrm{Gbit} / \mathrm{s}$ 混 传的能力。

第六, 系统应合理配置保护和恢复用的觉余波道, OTN
设备内部各子架应适当预留一定数量的业务槽位, 以备网络 调整等使用。

\section{3 波道配置原则}

4.3.1 按业务需求配置原则

依据各业务类型对带宽的实际需求进行配置, 将调度数据 网、通信数据网、MIS 网等大带宽需求的作为主要承载对象 ${ }^{[5]}$ 。

\subsection{2 波道合复用原则}

依据各业务在 OTN 网络上相关的路径, 按照每个波道 $10 \mathrm{G}$ 最大带宽的方式进行合复用, 确定波道实际的需求数量。

\section{4 典型配置}

OTN 站点设备典型配置包括电交叉子框、光子框、光放 大板、光监控信道板、光合波板、光分波板及公共部分等, 典型 配置原则如下: (1) OTN 公共部分: 按照 1+1 保护配置; (2)电交 叉子框: 各方向集中配置, 槽位不够时, 增加扩展子框; (3)光子 框: 每个方向配置 1 个; (4)光合波板/分波板: $\mathrm{M}$ 个方向数:每 个方向 80 波配 2 个, 40 波配 1 个; (5) OTU 端口数: 每个方向 按实际业务需求配置; (6)业务侧波道数: 每个方向按实际波道 需求配置; (7)支路板: 根据业务落地需求配置; (8)光放大板 $\mathrm{OAU} / \mathrm{OBU}$ : 每个方向配置, 数量按实际距离参数计算; (9)光监 控信道单元: 每个方向需配置一个监控信道; 10DCM 模块: 每 个方向, 距离超过 $40 \mathrm{~km}$ 应采用 DCM 补偿。

\section{5 结语}

智能电网的发展带动业务大容量带宽的需求, 采用先进 的 OTN 技术在电力系统组建时, 需要根据各地的通信网实际 状态, 以及结合各地的业务带宽需求, 综合选择合适的通信网 络规划, 同时必须结合现有的 SDH 网络, 将通信网网络构建 平滑的演进, 选择合适的方案, 以此来保证 OTN 技术能够充 分的发挥优势的作用, 保证电力通信网络能够在安全、稳定的 环境下运行,加快我们电力泛在互联网的建设速度。

\section{参考文献}

[1]金广祥.OTN 技术在电力通信系统中的应用研究[D]. 北京:华 北电力大学,2013.

[2]马岩.河北省南网大容量干线传输网工程方案设计 $[D]$. 北京: 华北电力大学, 2016 .

[3]齐聪, 鄢波.OTN 技术在江西电网的应用与规划[J].江西电力, 2017(12):43-45.

[4]李乐优.OTN 技术在电力通信系统中的应用与优化 $[\mathrm{D}]$. 大连: 大连理工大学, 2018

[5]马立新, 强晟, 赵亮, 等.OTN 承载平台在省级核心通信网络中 的规划与应用 $[J]$.电力信息与通信技术,2017(2):41-46. 